\title{
Curve Fitting Algorithm-Based Contrast-Enhanced Ultrasound Image for Evaluating the Effect of Dexmedetomidine on Neurological Function in Patients Undergoing Brain Tumor Surgery
}

\author{
Zhu Wu $\mathbb{D}$, Xue Sun $\mathbb{D}$, Yu Wu $\mathbb{D}$, and Haiying Zhang $\mathbb{D}$ \\ Department of Neurosurgery, First Affiliated Hospital of Soochow University, Suzhou 215000, Jiangsu, China \\ Correspondence should be addressed to Haiying Zhang; 201812210602032@zcmu.edu.cn
}

Received 12 December 2021; Revised 20 January 2022; Accepted 27 January 2022; Published 28 February 2022

Academic Editor: M Pallikonda Rajasekaran

Copyright $(92022$ Zhu Wu et al. This is an open access article distributed under the Creative Commons Attribution License, which permits unrestricted use, distribution, and reproduction in any medium, provided the original work is properly cited.

\begin{abstract}
Objective. The study aimed to explore the influence of dexmedetomidine (Dex) guided by ultrasonic contrast on postoperative cognitive function, serum nerve injury factors, inflammatory response, and blood glucose in patients undergoing intracerebral tumor resection through curve fitting algorithm. Methods. 80 patients who underwent tumor resection were selected and randomly rolled into control (Ctrl) group and Dex group, with 40 in each. Then, patients in the Dex group received $0.4 \mu \mathrm{g} / \mathrm{kg} \cdot \mathrm{h}$ Dex, and patients in Ctrl received the same amount of normal saline. Both groups were diagnosed with benign or malignant tumors by contrast-enhanced ultrasound (CEUS) based on time-intensity curve (TIC) before surgery. Cognitive Capacity Screening Examination (CCSE) and Montreal Cognitive Assessment (MoCA) were used to assess the cognitive function 1 day before and 3 days after the operation, and the ELISA method was used to detect levels of nerve damage-related factors and inflammation factor. Finally, the fasting blood glucose (FBG) levels were detected at the same time. Results. In contrast with benign lesions, the AUC, MTT, and PI of malignant lesions were obviously reduced $(P<0.05)$, while TP was obviously increased $(P<0.05)$. The postoperative CCSE and MoCA scores of the Dex group were obviously higher than Ctrl $(P<0.05)$. In contrast with the Ctrl, the CCSE and MoCA scores in the Dex group increased obviously after 3 days $(P<0.05)$. In addition, the incidence of postoperative cognitive dysfunction (POCD) in the Dex group was obviously reduced $(P<0.05)$. Besides, levels of S100 $\beta$, NSE, and GFAP were obviously reduced in the Dex group 1 day after surgery $(P<0.05)$, while levels of IL- 6 and TNF- $\alpha$ were obviously reduced $(P<0.05)$, and returned to the level of Ctrl 3 days after surgery. There was no obvious difference in FBG at each time point between the two. Conclusion. CEUS based on curve fitting algorithm is effective on nursing treatment of intracranial tumors. Dex can obviously improve POCD and reduce levels of serum nerve injury factors and inflammatory factors.
\end{abstract}

\section{Introduction}

Brain tumor is a relatively common neurological disease in clinical practice. Because the compression of tumor will cause increased intracranial pressure, there will also be invasive operation during surgery causing hemodynamic changes and brain tissue hypoxia and ischemia, which will lead to irreversible damage to the neurological function and cognitive function $[1,2]$. On the other hand, postoperative cognitive dysfunction (POCD) is also a possible postoperative complication in patients undergoing general anesthesia surgery, causing memory loss, reduced living ability, psychological abnormalities, and decreased orientation in patients [3]. POCD is the main cause of increased mortality, delayed recovery, accidental complications, and prolonged hospital stay $[4,5]$. Dexmedetomidine (Dex) is an anesthetic widely used in clinical practice, which has analgesic and sedative effects, has little effect on hemodynamics, and can reduce the damage of surgery and anesthetic drugs to brain tissue to a certain extent and play a role in cerebral protection $[6,7]$. It is well known that Dex has the similar nursing effect of opioids, and these characteristics make it a 
suitable choice for sedatives in intensive care unit (ICU) and perioperative conditions [8].

CEUS is a common method for the diagnosis of intracranial tumors. Through intravenous injection of contrast agent, the microbubble components can enter the blood circulation. Because the microbubbles have sound scattering properties, the acoustic impedance between the target location and the surrounding tissues can be formed. When the microbubble component of the contrast agent reaches the lesion tissue, the microcirculation blood perfusion state of the tissue can be objectively judged from the number and rate of its ingress and egress. As a result, the lesion tissue can form an image that is different from the normal tissue, with diagnostic significance [9]. The contrast-enhanced signal changes with time. This process is refitted to a time-intensity curve. This curve can be used to quantitatively analyze the blood flow characteristics of the lesion tissue, which is of great significance for the differential diagnosis of tumors.

Curve fitting algorithm-based CEUS was adopted to diagnose benign and malignant tumors in patients undergoing intracranial tumor resection. Then, the effects were explored of Dex anesthesia nursing on patients' cognitive function, nerve injury factors, inflammatory factors, and blood glucose before and after surgery. It is expected to provide experimental evidence for the feasibility of Dex in patients with intracranial tumors during the perioperative period and scientific evidence for Dex to improve POCD.

\section{Materials and Methods}

2.1. Research Subjects. Eighty patients who underwent tumor resection in hospital from January 2019 to January 2020 were selected as research subjects, including 44 males and 36 females. They were between 53 and 65 years old, with an average age of $(57.85 \pm 6.94)$ years. The control group (Ctrl) had 40 patients, including 19 males and 21 females, aged $50-61$ years, with a mean age of $(60.16 \pm 7.2)$ years; the Dex group had 40 patients, including 25 males and 15 females, aged $55-65$ years, with a mean age of $(61.09 \pm 6.9)$ years. This study had been approved by the Ethics Committee of hospital, and all the study objects and their families were informed and voluntarily agree to participate in this study.

Inclusion criteria: patients who planned to undergo supratentorial tumor resection; preoperative MRI imaging indicated that the diameter of the tumor was less than or equal to $40 \mathrm{~mm}$; education level was above high school; the preoperative cognitive capacity screening examination (CCSE) score was $\geq 20$ points; all patients and their families had signed informed consent forms. Exclusion criteria: patients with a history of craniotomy; patients with severe cardiopulmonary insufficiency and other organic diseases; pregnant or lactating female patients; patients with a history of mental illness and a history of long-term use of sedative drugs; and patients involved in this clinical trial.

2.2. CEUS Based on Curve Fitting Algorithm. All patients underwent CEUS to assess the degree of benign and malignant intracranial tumors. The ultrasonic diagnostic was used. The L12-5 (5MHz-12 MHz) probe was adopted for routine inspections, and the $\mathrm{L} 9-3(3 \mathrm{MHz}-9 \mathrm{MHz})$ probe was used to observe the contrast of intracranial tumors. The main component of the contrast agent was sulfur hexafluoride coated with phospholipids. During the examination, the patient was placed in a supine position, the probe was placed $2.5-3.0 \mathrm{~cm}$ above the external auditory canal hole on the ear side. It can be moved forward and backward. The L12-5 probe was used to observe the size, position, boundary, echo, and calcification of the tumor. After the target lesion was determined, the L9-3 probe was adopted to observe the overall view of the lesion, and the best angiography section was selected and the probe was fixed. The angiography conditions such as gain and mechanical index were consistent in the angiography mode. After $2.4 \mathrm{~mL}$ of contrast agent was injected through the cubital vein, $10 \mathrm{~mL}$ of $0.9 \%$ sodium chloride solution was quickly followed. At the same time, the $120 \mathrm{~S}$ dynamic image was collected. Philips QLAB9.1 was adopted to analyze the contrast results of the lesion area and the nonlesion area. The change of signal intensity over time was analyzed. Besides, a TIC was obtained. LDRW-WIWO was used to get curve fitting-ultrasound mean value. Then, the following 4 clinical parameters were obtained: area under curve (AUC), rise time (TP), mean channel time (MTT), and peak intensity (PI). All CEUS examinations and diagnosis processes were completed by 3 clinicians with more than 3 years of work experience.

2.3. Grouping and Intervention Methods. All patients were randomly rolled into Ctrl and Dex groups, with 40 cases in each group. They were subjected to routine fasting and water prohibition before surgery, and medication was avoided before surgery. During surgery, Dex group received continuous intravenous infusion of $0.4 \mu \mathrm{g} / \mathrm{kg} \cdot \mathrm{h} \mathrm{Dex}$, and Ctrl received the same amount of normal saline until the operation was completed. At the same time, the anesthesia induction and anesthesia maintenance protocols of the two were the same, and target-controlled infusion of propofol and remifentanil were performed on both. During the operation, the patient's blood pressure, end-tidal carbon dioxide, electrocardiogram, bispectral index (BIS), and pulse oxygen saturation were monitored in real time. 30 minutes before the completion of the operation, $50 \mathrm{mg}$ flurbiprofen axetil was injected intravenously for postoperative analgesia, and $5 \mathrm{mg}$ tropisetron was given to prevent postoperative nausea and vomiting.

2.4. Assessment of Cognitive Function. CCSE and MoCA were used to evaluate the cognitive function and the incidence of POCD 1 day before and 3 days after operation. The CCSE assessed cognitive function mainly from several aspects such as orientation, attention, instantaneous memory, recall, language, and mental arithmetic ability, with a maximum score of 30 points. The score range was between 0 and 30 points. The score was positively correlated with the cognitive function. MoCA can evaluate the cognitive function of patients from 8 aspects including memory, 
attention, visual space executive function, language, delayed recall, naming, orientation, and abstract thinking ability. The score range was between 0 and 30 points. The score was positively correlated with the cognitive function.

2.5. Detection of Serum Nerve Injury-Related Factors and Inflammatory Response Factors. The ELISA method was used to detect serum nerve injury-related factors, including S100 $\beta$, neuron-specific enolase (NSE), and glial fibrillary acidic protein (GFAP), 1 day before operation and 3 days after operation, and inflammatory response factors, including IL- 6 and TNF- $\alpha$. The serum samples were obtained at the same time point for the two. At the same time, the FBG levels were detected 1 day before the operation and 3 days after the operation. The blood glucose meter was provided by Roche.

2.6. Statistical Analysis. All data were processed by SPSS22.0. The mean \pm standard deviation was how the measurement was expressed, and the comparison between groups was by $t$-test. The enumeration data were expressed by the number of cases (percentage), and the chi-square test was used for comparison between groups. $P<0.05$ indicated that the difference was statistically significant.

\section{Results}

3.1. Ultrasonic Image. Figure 1 is an ultrasonographic image of a 62-year-old patient with brain tumor, and it shows ring enhancement with clearly visible borders under contrastenhanced ultrasound. Figure B shows ring enhancement under contrast-enhanced ultrasound with visible internal necrotic areas. During the operation, all patients were able to achieve maximum extent of tumor resection under ultrasound guidance.

3.2. Quantitative Analysis of TIC. The comparative analysis of TIC parameters of benign and malignant lesions and their corresponding para-carcinoma tissues is shown in Figures 2 and 3. In contrast with benign lesions, the AUC, MTT, and PI of malignant lesions were obviously reduced $(P<0.05)$. The TP increased obviously $(P<0.05)$. The AUC, MTT, and PI of malignant lesions were obviously lower than the adjacent tissues $(P<0.05)$, and the TP was obviously higher than the adjacent tissues $(P<0.05)$.

3.3. Comparison of Preoperative and Postoperative Cognitive Function. The comparative analysis of the CCSE and MoCA scores 1 day before and 3 days after the operation was shown in Figure 4. The CCSE and MoCA scores of the Dex group after the operation were obviously higher than $\operatorname{Ctrl}(P<0.05)$. The preoperative CCSE and MoCA scores of the Ctrl were obviously higher than those after the operation $(P<0.05)$.

3.4. POCD Incidence Rate 3 Days after Surgery. The comparative analysis of the incidence of POCD 3 days after surgery is shown in Figure 5. Based on the CCSE score, the incidence of POCD in the Dex group was obviously lower than that in the Ctrl $(P<0.05)$. Similarly, based on the MoCA score, the incidence of POCD in the Dex group was obviously lower than $\mathrm{Ctrl}(P<0.05)$.

3.5. Comparison of the Levels of Nerve Injury-Related Factors. The comparison and analysis of the levels of S100 $\beta$, NSE, and GFAP at 1 day before operation, 1 day after operation, and 3 days after operation are shown in Figures 6 and 7. There was no difference in the levels of these factors. The levels of these in the Dex group were obviously lower than those in the Ctrl group at 1 day after surgery $(P<0.05)$. The levels of these in the two groups on the 1st and 3rd days after the operation were obviously higher than those on 1 day before the operation $(P<0.05)$.

3.6. Comparison of the Levels of Inflammatory Response Factors. The comparison and analysis of the levels of IL-6 and TNF- $\alpha$ at 1 day before operation, 1 day after operation, and 3 days after operation are shown in Figure 8. There was no obvious difference in the levels of these two before operation. The levels of the two in the Dex group were obviously lower than the Ctrl group at 1 day after surgery $(P<0.05)$. On the 1 st and $3 r d$ days after the operation, the levels of the two were obviously higher than those on the 1st day before the operation $(P<0.05)$.

3.7. Comparison of Blood Glucose Levels. The comparative analysis of the FBG levels 1 day before operation, 1 day after operation, and 3 days after operation is shown in Figure 9. There was no obvious difference in the FBG levels 1 day before operation, 1 day after operation, and 3 days after operation.

\section{Discussion}

CEUS can monitor the perfusion state of the tiny blood flow in the tumor in real time, thereby improving the sensitivity and specificity of tumor lesion monitoring, and Dex has the nursing effect of analgesia during operation. The trend and quantitative analysis index of TIC can objectively reflect the characteristics of filling and disappearing of the contrast agent over time in the imaging process [10]. PI can reflect the total amount of microbubbles flowing into the nodules, and TP and MTT can reflect the filling and regression speed of the microbubbles [11]. In this study, CEUS was used to observe the blood perfusion pattern of intracranial tumors, and TIC was used for curve simulation quantitative analysis. It was found that, in contrast with benign lesions, the AUC, MTT, and PI of malignant lesions were obviously reduced, while TP was obviously increased. The AUC, MTT, and PI of malignant lesions were obviously lower than their adjacent tissues, while TP was obviously higher than their adjacent tissues. It can be inferred that TIC parameters were indicative in diagnosing benign and malignant lesions. The TIC parameters of benign lesions were similar to those of adjacent tissues, while the TIC parameters of malignant lesions 


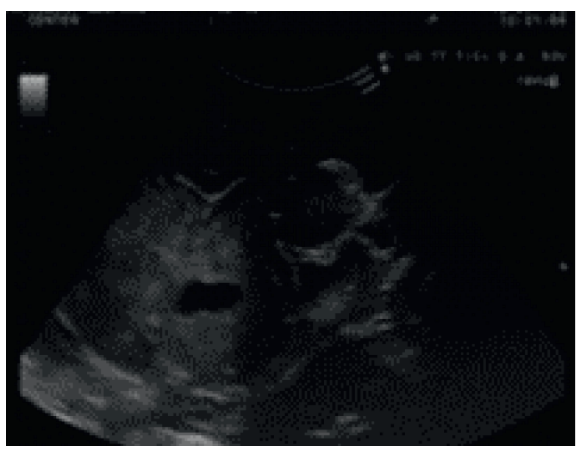

(a)

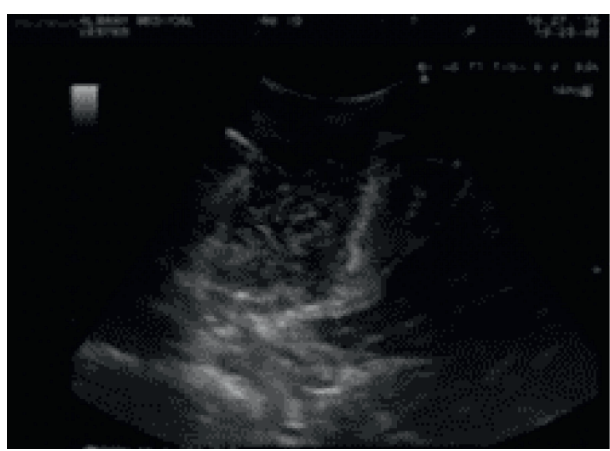

(b)

FIGURE 1: Ultrasonic image.

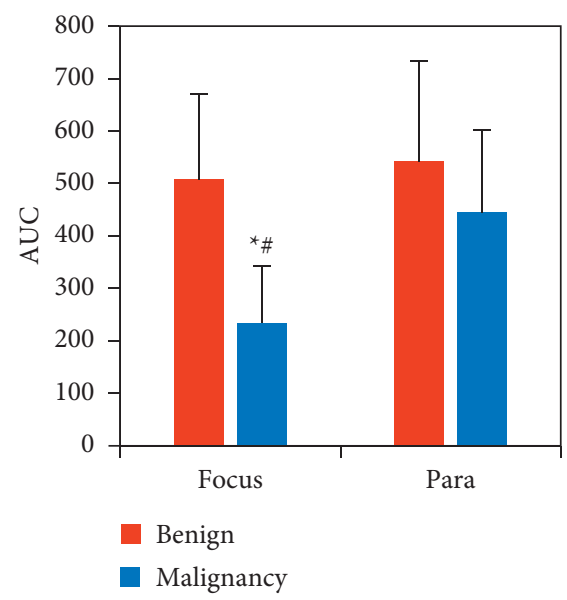

(a)

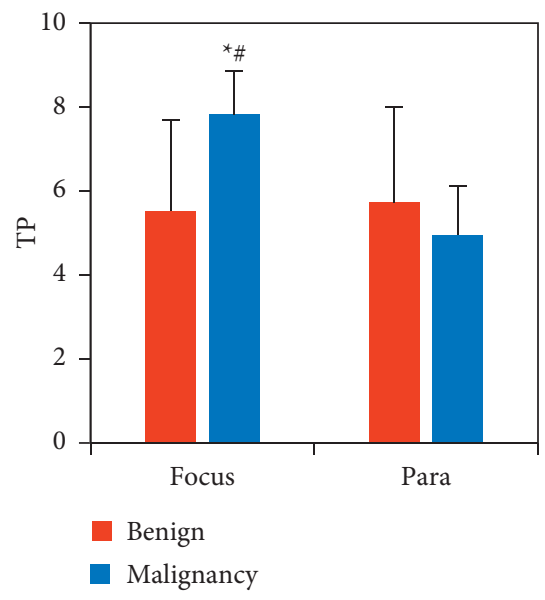

(b)

FIGURe 2: Quantitative analysis of TIC parameters. (a) AUC and (b) TP. ${ }^{*}$ indicates statistically significant difference compared with benign lesions, $P<0.05$; \# indicates statistically significant difference compared with adjacent tissues, $P<0.05$.

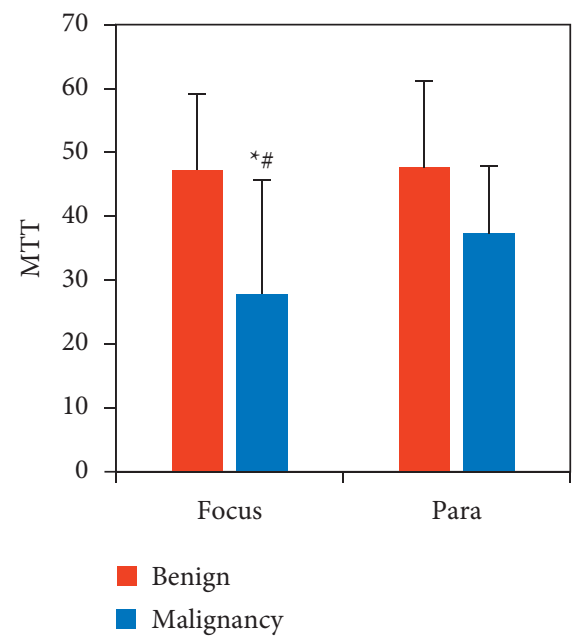

(a)

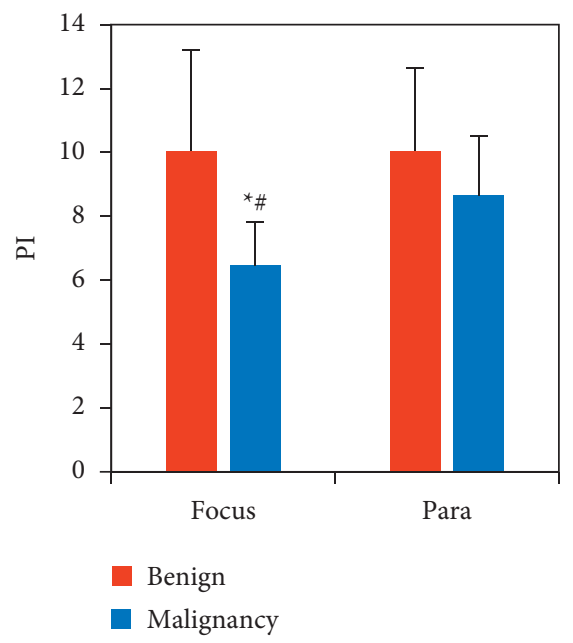

(b)

FIgUre 3: Quantitative analysis of TIC parameters. (a) MTT. (b) PI. * indicates statistically significant difference compared with benign lesions, $P<0.05$; \# indicates statistically significant difference compared with adjacent tissues, $P<0.05$. 


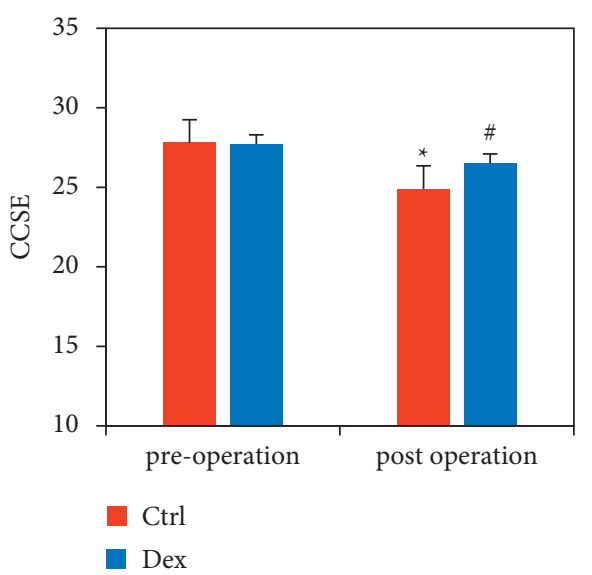

(a)

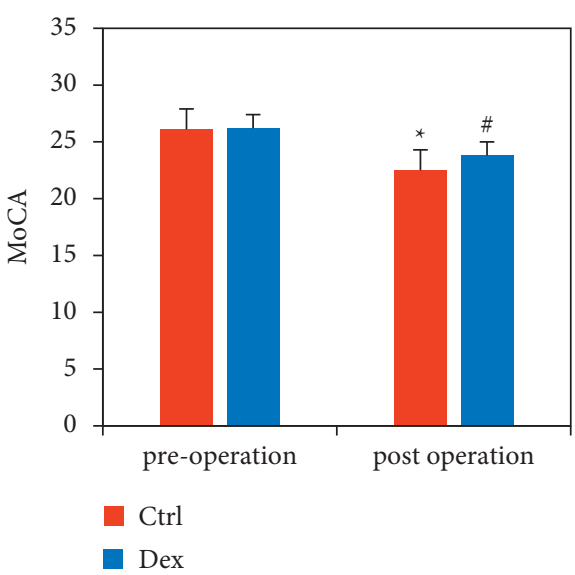

(b)

Figure 4: Comparison of preoperative and postoperative cognitive function. (a) CCSE score. (b) MoCA score. ${ }^{*} P<0.05$, in contrast with preoperative; $\# P<0.05$, in contrast with Ctrl.

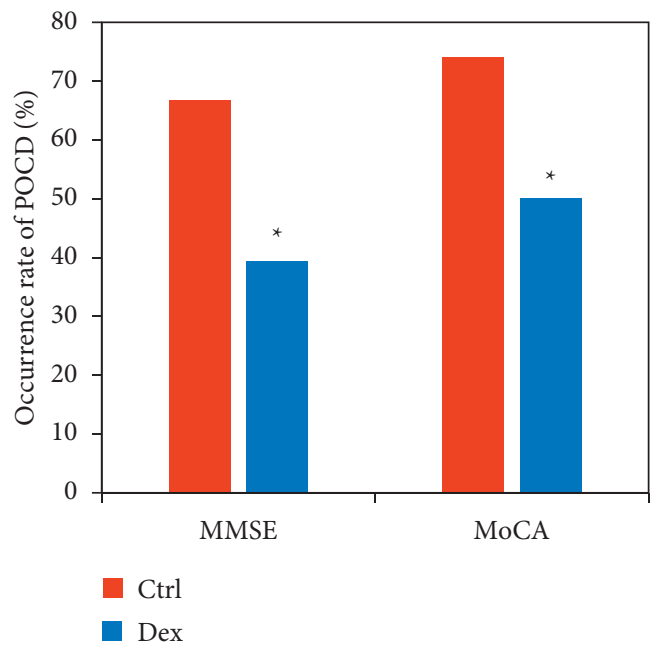

FIgUre 5: Comparison of preoperative and postoperative POCD incidence. ${ }^{*} P<0.05$, in contrast with Ctrl.

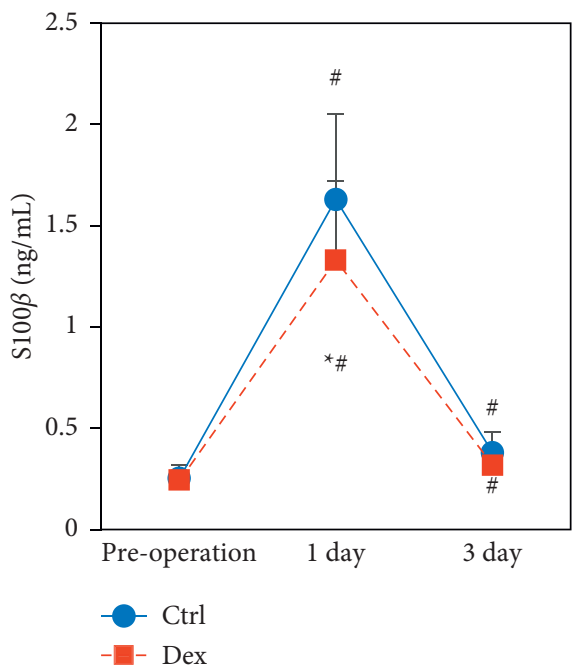

(a)

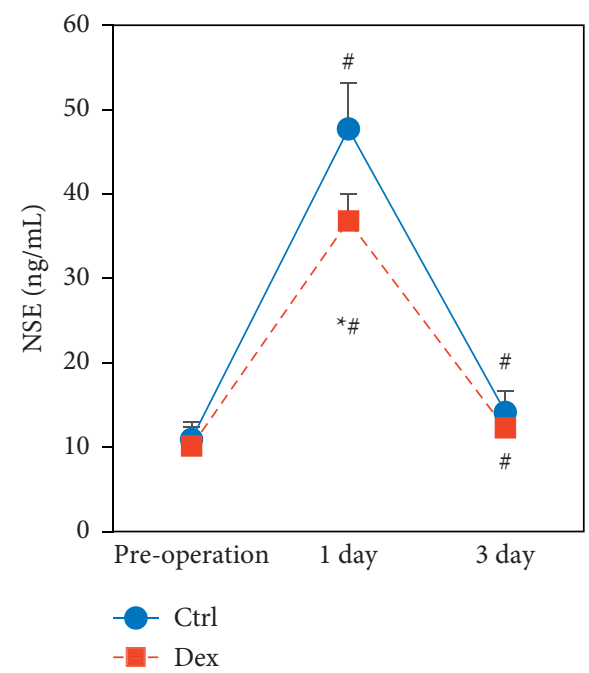

(b)

Figure 6: Comparison of $S 100 \beta$ and NSE levels. ${ }^{*} P<0.05$, in contrast with Ctrl; $\# P<0.05$, in contrast with preoperative. 


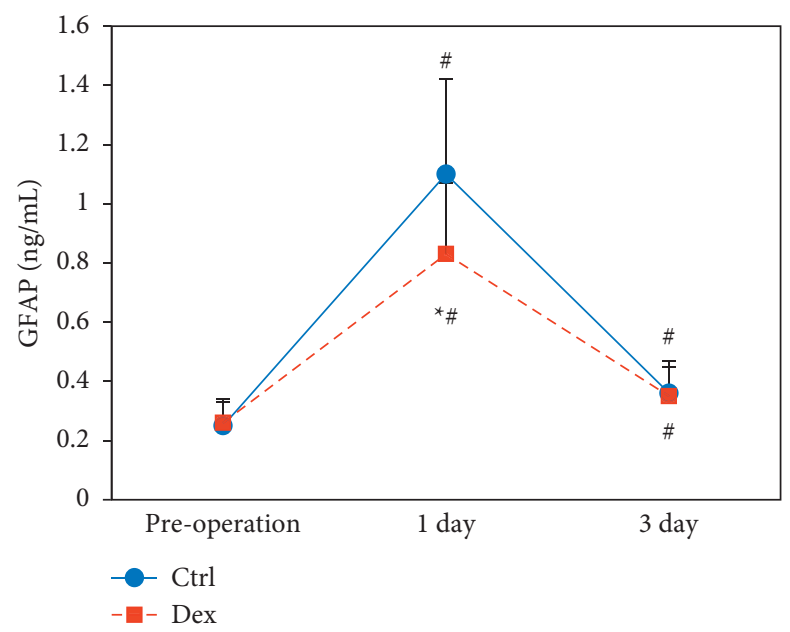

FIgURe 7: Comparison of preoperative and postoperative GFAP levels. ${ }^{*} P<0.05$, in contrast with Ctrl; $\# P<0.05$, in contrast with preoperative.

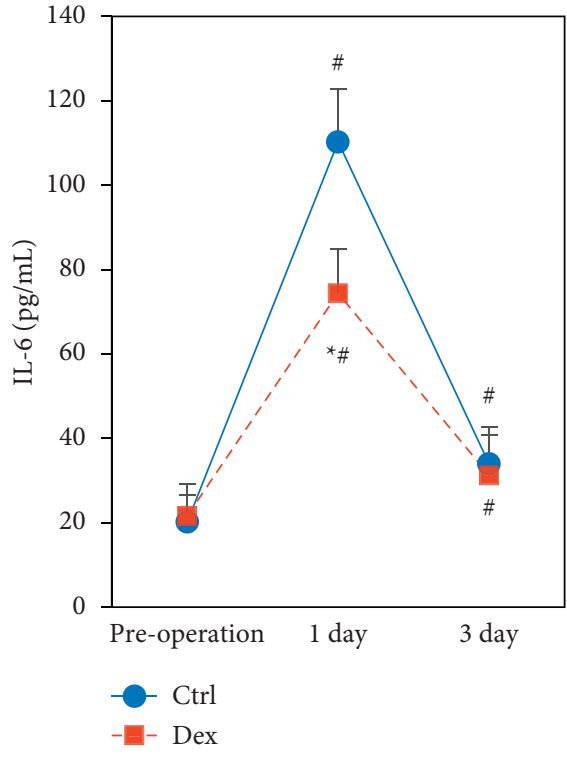

(a)

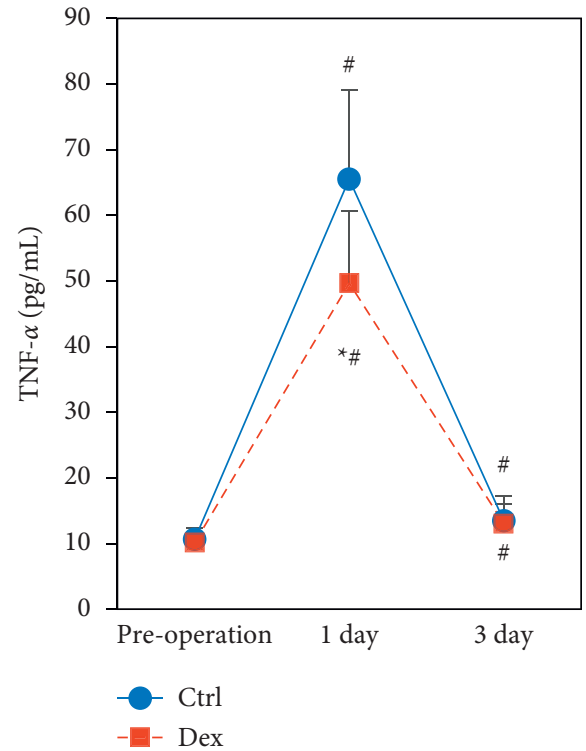

(b)

Figure 8: Comparison of IL-6 and TNF- $\alpha$ levels. ${ }^{*} P<0.05$, in contrast with Ctrl; $\# P<0.05$, in contrast with preoperative.

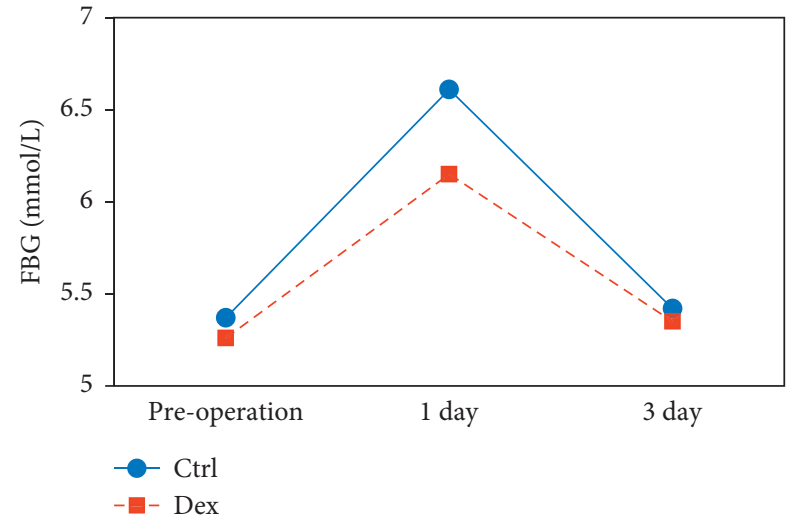

Figure 9: Comparison of FBG levels. are different from those of adjacent tissues and benign lesions, which was consistent with previous studies [12].

Studies have shown that Dex can significantly improve CCSE score 1 day after anesthesia for patients with meningioma [13]. In patients with ischemic cerebrovascular disease, $1 \mu \mathrm{g} / \mathrm{kg}$ Dex anesthesia was used during surgery, and it was found that it can obviously improve the MoCA score of 3 days after surgery, the patient's attention and delayed recall, and restore the patient's cognition function [14]. It was consistent with the results in this study. The CCSE and the MoCA were adopted to assess the effect of Dex on postoperative cognitive function. It was found that there was no obvious difference in the CCSE and MoCA scores. The two scores of the Dex group were obviously higher than those of the Ctrl. At the same time, based on the two scales, 
the incidence of postoperative POCD was judged. This, in the Dex group, was obviously lower than that in the Ctrl. It was consistent with previous studies of meningioma patients and patients with ischemic cerebrovascular disease [15], indicating that continuous intraoperative Dex infusion can obviously improve postoperative cognitive function and reduce the incidence of postoperative POCD.

$S 100 \beta$ protein is mainly distributed in the central nervous system and is a kind of acidic calcium binding protein, mainly synthesized and secreted by astrocytes. When the brain is injured, the cell membrane of astrocytes is destroyed and the blood-brain barrier is damaged, causing S100 $\beta$ to enter the cerebrospinal fluid or blood from astrocytes. As a result, the serum S100 $\beta$ level increases obviously [16]. NSE exists in neurons and neuroendocrine cells. GFAP is a specific marker of astrocytes. When brain injury occurs, the blood-brain barrier is destroyed, and NSE and GFAP enter the cerebrospinal fluid and blood, resulting in increased serum NSE and GFAP levels [17-19]. It was found that there was no obvious difference in the levels of $S 100 \beta$, NSE, and GFAP before surgery. The levels of these in the Dex group were obviously lower than those in the Ctrl at 1 day after surgery. There was no obvious difference in the levels of these at 3 days after surgery. The levels of these were obviously higher at 1 day after surgery and 3 days after surgery than 1 day before surgery, but there was no obvious difference in the levels of these at 1 day and 3 days after surgery, indicating that Dex can obviously promote the restoration of nerve injury factors to baseline levels, repair the function of neurons and glial cells, and then protect the brain. In addition, inflammation of the central nervous system is an important mechanism leading to perioperative brain injury. The proinflammatory factors are important factors involved in the perioperative inflammatory response, and the increase in their levels in the serum indicates an increase in the degree of systemic inflammation. The results in the study revealed that there was no obvious difference in the levels of IL- 6 and TNF- $\alpha$ before surgery. The levels of the two at 1 day and 3 days after the operation were obviously higher than those at 1 day before the operation, but there was no obvious difference in the levels of the two at 1 day and 3 days after the operation. It was consistent with the results of Meng et al. [20], but it showed that Dex had no significant effect on perioperative blood glucose levels in patients.

\section{Conclusion}

CEUS based on curve fitting algorithm is effective on the diagnosis of intracranial tumors. At the same time, continuous intraoperative Dex infusion for anesthesia nursing can obviously improve the postoperative cognitive function, reduce the incidence of postoperative POCD, and protect the brain. The mechanism may be related to the levels of Dex promoting nerve injury factors and inflammation factors. However, benign lesions and malignant lesions have not been evaluated based on the results of CEUS, after which grouped interventions of Dex should be performed separately. The problem is caused by time constraints. In conclusion, this study can still provide an experimental basis for the feasibility of Dex in patients with intracranial tumors during the perioperative period and a scientific basis for postoperative cognitive function nursing.

\section{Data Availability}

The data used to support the findings of this study are available from the corresponding author upon request.

\section{Conflicts of Interest}

The authors declare no conflicts of interest.

\section{Acknowledgments}

This work was supported by Suzhou City Science and Education to Revitalize Weiqing year of science and technology project (no. KJXW2017006).

\section{References}

[1] N. Kotekar, A. Shenkar, and R. Nagaraj, "Postoperative cognitive dysfunction - current preventive strategies," Clinical Interventions in Aging, vol. 13, pp. 2267-2273, 2018.

[2] M. J. Needham, C. E. Webb, and D. C. Bryden, "Postoperative cognitive dysfunction and dementia: what we need to know and do," British Journal of Anaesthesia, vol. 119, no. suppl_1, pp. i115-i125, 2017.

[3] L. A. Daiello, A. M. Racine, R. Yun Gou et al., "Postoperative delirium and postoperative cognitive dysfunction," Anesthesiology, vol. 131, no. 3, pp. 477-491, 2019.

[4] Y. L. Chi, Z. S. Li, C. S. Lin, Q Wang, and Y. K Zhou, "Evaluation of the postoperative cognitive dysfunction in elderly patients with general anesthesia," European Review for Medical and Pharmacological Sciences, vol. 21, no. 6, pp. 1346-1354, 2017.

[5] A. Schnabel, S. U. Reichl, S. Weibel et al., "Efficacy and safety of dexmedetomidine in peripheral nerve blocks," European Journal of Anaesthesiology, vol. 35, no. 10, pp. 745-758, 2018.

[6] K.-M. Chiu, T.-Y. Lin, M.-Y. Lee, C.-W. Lu, M.-J. Wang, and S.-J. Wang, "Dexmedetomidine protects neurons from kainic acid-induced excitotoxicity by activating BDNF signaling," Neurochemistry International, vol. 129, Article ID 104493, 2019.

[7] Z. Y. Yu, J. Geng, Z. Q. Li et al., "Dexmedetomidine enhances ropivacaine-induced sciatic nerve injury in diabetic rats," British Journal of Anaesthesia, vol. 122, no. 1, pp. 141-149, 2019.

[8] X. L. Wang, J. Wang, D. L. Mu, and D. X. Wang, “[Dexmedetomidine combined with ropivacaine for continuous femoral nerve block improved postoperative sleep quality in elderly patients after total knee arthroplasty]," Zhonghua Yixue Zazhi, vol. 98, no. 10, pp. 728-732, 2018.

[9] E. Ilunga-Mbuyamba, J. G. Avina-Cervantes, D. Lindner, I. Cruz-Aceves, F. Arlt, and C. Chalopin, "Vascular structure identification in intraoperative 3D contrast-enhanced ultrasound data," Sensors, vol. 16, no. 4, 2016.

[10] E. Gong, J. M. Pauly, M. Wintermark, and G. Zaharchuk, "Deep learning enables reduced gadolinium dose for contrastenhanced brain MRI," Journal of Magnetic Resonance Imaging, vol. 48, no. 2, pp. 330-340, 2018.

[11] L. G. Cheng, W. He, H. X. Zhang et al., "Intraoperative contrast enhanced ultrasound evaluates the grade of glioma," 
BioMed Research International, vol. 2016, Article ID 2643862, 9 pages, 2016.

[12] J. Novak, S. B. Withey, S. Lateef, L. MacPherson, B. Pinkey, and A. C. Peet, "A comparison of pseudo-continuous arterial spin labelling and dynamic susceptibility contrast MRI with and without contrast agent leakage correction in paediatric brain tumours," British Journal of Radiology, vol. 92, no. 1094, Article ID 20170872, 2019.

[13] H. Zhang, Z. Wu, X. Zhao, and Y Qiao, "Role of dexmedetomidine in reducing the incidence of postoperative cognitive dysfunction caused by sevoflurane inhalation anesthesia in elderly patients with esophageal carcinoma," Journal of Cancer Research and Therapeutics, vol. 14, no. 7, pp. 14971502, 2018.

[14] M. Muzzi, D. Buonvicino, M. Urru, L. Tofani, and A. Chiarugi, "Repurposing of dexpramipexole to treatment of neonatal hypoxic/ischemic encephalopathy," Neuroscience Letters, vol. 687, pp. 234-240, 2018.

[15] L. Teng, W. Chen, C. Yin, H. Zhang, and Q. Zhao, "Dexmedetomidine improves cerebral ischemia-reperfusion injury in rats via extracellular signal-regulated kinase/cyclic adenosine monophosphate response element binding protein signaling pathway," World Neurosurgery, vol. 127, pp. e624e630, 2019.

[16] H. Lv, Q. Wang, S. Wu et al., "Neonatal hypoxic ischemic encephalopathy-related biomarkers in serum and cerebrospinal fluid," Clinica Chimica Acta, vol. 450, pp. 282-297, 2015.

[17] D. V. Agoston, A. Shutes-David, and E. R. Peskind, "Biofluid biomarkers of traumatic brain injury," Brain Injury, vol. 31, no. 9, pp. 1195-1203, 2017.

[18] C. Han, R. Fu, and W. Lei, "Beneficial effects of dexmedetomidine on early postoperative cognitive dysfunction in pediatric patients with tonsillectomy," Experimental and Therapeutic Medicine, vol. 16, no. 1, pp. 420-426, 2018.

[19] S. Xun and R. Zheng, "Retracted: dexmedetomidine alleviates neuropathic pain by regulating JAK/STAT pathway in rats," Journal of Cellular Biochemistry, vol. 121, no. 3, pp. 22772283, 2020.

[20] L. Meng, L. Li, S. Lu et al., "The protective effect of dexmedetomidine on LPS-induced acute lung injury through the HMGB1-mediated TLR4/NF- $\kappa$ B and PI3K/Akt/mTOR pathways," Molecular Immunology, vol. 94, pp. 7-17, 2018. 